\title{
Editorial: Beneficial Microbes and the Interconnection Between Crop Mineral Nutrition and Induced Systemic Resistance
}

\author{
Carlos Lucena ${ }^{1 *}$, Sabine Dagmar Zimmermann ${ }^{2 *}$, Jianfei Wang ${ }^{3 *}$ and Ricardo Aroca ${ }^{4 *}$ \\ ' Departamento de Agronomía (DAUCO-María de Maeztu Unit of Excellence), Universidad de Córdoba, Córdoba, Spain, \\ ${ }^{2}$ BPMP, CNRS, INRAE, Institut Agro, Université de Montpellier, Montpellier, France, ${ }^{3}$ Anhui University of Science and \\ Technology, Huainan, China, ${ }^{4}$ Departamento de Microbiología del Suelo y Sistemas Simbióticos, Estación Experimental del \\ Zaidin (CSIC), Granada, Spain
}

Keywords: nutrient deficiency, ISR eliciting microbes, crops, soil, microbial consortia

\section{Editorial on the Research Topic}

Beneficial Microbes and the Interconnection Between Crop Mineral Nutrition and Induced Systemic Resistance

\section{OPEN ACCESS}

Edited and reviewed by: Jonathan Michael Plett, Western Sydney University, Australia

*Correspondence:

Carlos Lucena

b42/ulec@uco.es

Sabine Dagmar Zimmermann sabine.zimmermann@cnrs.fr Jianfei Wang

jfwang1@aliyun.com Ricardo Aroca

raroca@eez.csic.es

Specialty section: This article was submitted to Plant Symbiotic Interactions,

a section of the journal

Frontiers in Plant Science

Received: 07 October 2021 Accepted: 05 November 2021 Published: 29 November 2021

Citation:

Lucena C, Zimmermann SD, Wang $J$ and Aroca $R$ (2021) Editorial: Beneficial Microbes and the Interconnection Between Crop Mineral Nutrition and Induced Systemic Resistance.

Front. Plant Sci. 12:790616. doi: 10.3389/fp/s.2021.790616
To cope with nutrient deficiencies, plants develop morphological and physiological responses, mainly in their roots, aimed to facilitate nutrient acquisition (Lucena et al., 2018). In the last years, it has been found that some rhizosphere microbes can induce physiological and morphological responses in roots of dicot plants similar to the ones induced by plants under nutrient deficiencies (Verbon et al., 2017). Remarkably, these rhizosphere microbes are also capable of eliciting the induced systemic resistance (ISR) against pathogens and insects (Pieterse et al., 2014; Verbon et al., 2017). This observation suggests that both processes (ISR and nutrient deficiency responses) are closely interconnected thus opening new possibilities for optimizing the management of the rhizosphere microbiota for improving mineral nutrition and health (Zamioudis et al., 2015; Verbon et al., 2017, 2019). However, the nodes of convergence between the two processes remain unclear (Romera et al., 2019). Elucidating the main nodes of interconnection between the pathways regulating microbe-elicited ISR and mineral uptake is critical for optimizing the use of plant mutualistic microbes in agriculture. The Research Topic updates latest findings related to the roles of ISR eliciting microbes in crops. It includes 12 original articles and one review, eight articles are related to beneficial microbes as biocontrol effectors inducing disease resistance and growth promotion of their hosts (Cueva-Yesquén et al.; La Spada et al.; Qu et al.; Qin et al.; Tseng et al.; Yu et al.; Zhou et al.; Zhu et al.), two articles concern growth promotion under abiotic stress (Tseng et al.; Yuan et al.), two others are linked to reduced chemical fertilization or soil property changes (Cardoso et al.; Wang et al.), and one to the role of $\mathrm{N}_{2}$ fixing bacteria (Kordi et al.). The review is related to the special role of mycorrhizal fungi on orchid seed germination (Zhao et al.).

Regarding the role of diverse microorganisms as biocontrol effectors, a variability of physiological and molecular mechanisms has been observed. The tight link between beneficial effects of microorganisms on plant growth by improved nutrition and defense priming through systematic enhancement of resistance against below-ground and above pathogens or insect herbivores has been described for arbuscular mycorrhizal (AM) fungi as mycorrhiza-induced resistance (MIR) (Cameron et al., 2013). Such interactions might be dependent on abiotic factors and mediated by jasmonic acid (JA) signaling. This interconnection between Plantago lanceolate with the AM fungus Funneliformis mosseae and the herbivore Mamestra brassica was studied by 
$\mathrm{Qu}$ et al.. Surprisingly, in contrast to tomato (Rivero et al., 2021), they reported in their specific case a repression of JAmediated defense by AM fungi, underlining the complexity of the studied model under their selected conditions (symbiotic and pathogen partners, age of plants, light, soil P, JA treatments). Mycoparasitic Trichoderma fungi have been used as biocontrol agents (Guzmán-Guzmán et al., 2019). La Spada et al. demonstrated that two selected Trichoderma strains (T. asperellum and $T$. atroviride) promoted tomato growth and reduced the disease severity caused by the oomycete Phytophthora nicotianae. Genetic patterns of the components of the experimental model tomato-Trichoderma spp.-P. nicotianae were differentially modified. Both counteract the challenge of infections by modulating the expression of crinkler, necrosisinducing Phytophthora protein 1, and cellulose-binding elicitor lectin pathogenic effectors involved in plant defense mechanisms. Tseng et al. isolated a new endophytic fungus (a Trichoderma strain) from the leaves of a deciduous wood tree Leucas aspera. When applied to Arabidopsis thaliana and Nicotiana attenuata, this fungus colonized their roots thereby strongly promoting the initial plant growth in soil. The fungus showed predatory capability on the pathogenic fungus Alternaria brassicicola. Colonized A. thaliana plants displayed lower A. brassicicola spread in roots and shoots, while AM formation in N. attenuata was not affected by the Trichoderma strain.

Plant growth promoting bacteria (PGPB) living as endophytes display several beneficial traits as improving nutrient bioavailability, interfering with hormone levels, or protection against abiotic and biotic stress (de Souza et al., 2015; Kumar et al., 2020) thus leading also to better plant growth, development, and resistance. Cueva-Yesquén et al. isolated and analyzed such culturable bacterial endophytes from passionflower (Passiflora incarnata) by phenotypic and genotypic approaches and confirmed finally the probiotic effect of some of them by evaluating their capacity to boost germination and growth of another plant, namely of the Cape gooseberry (Physalis peruviana). In another study, in the context of rhizobacteria-mediated defense, Zhu et al. reported that the PGPB Pseudomonas fluorescens could increase the resistance of cucumber plants against infection by Botrytis cinerea. Pseudomonas bacteria have been used before as biocontrol effectors in different plant species (Kupferschmied et al., 2013; De Vrieze et al., 2020) and different mechanisms were proposed. Here, the authors found by RNA-sequencing that the improved defense would be linked to the expression of polyamine-associated and defense-related genes. Zhou et al. demonstrated the ability of the rhizobacterial strain Bacillus subtilis SL18r to trigger ISR in tomato plants against the foliar pathogen Botrytis cinerea. The authors reported that the long non-coding RNAs (lncRNAs) were involved in the mediation of the rhizobacteria-primed ISR processes in plants by a comparative transcriptome analysis between non-inoculated and SL18r-inoculated plants. Postharvest strawberry is susceptible to gray mold disease caused by $B$. cinerea. Yu et al. found that inoculation with Bacillus cereus diminished disease severity by modulating salicylic acid (SA) pathway as revealed by transcriptomic analysis, and enhancing antioxidant activity of strawberry fruits. Finally, the cotton seedling response to Bacillus circulans GN03 was explored by Qin et al. showing a remarkably enhanced growth promotion as well as disease resistance. GN03 inoculation altered the microbiota in and around the plant roots. At the physiological and molecular level, the authors observed a significant accumulation of growthrelated (indole acetic acid (IAA), gibberellic acid (GA), and brassinosteroids) and disease resistance-related hormones (SA, JA), an up-regulated expression of phytohormone synthesisrelated genes (EDS1, AOC1, BES1, GA20ox), of an auxin transporter gene (Aux1), and of disease-resistance genes (NPR1, PR1).

Regarding abiotic stress, it is well-known that PGPB enhance salt tolerance of plants by several mechanisms (Kumar et al., 2020), one of them might be the production of some organic compounds. One of these compounds are phenazines, a class of diffusible, heterocyclic compounds harboring substitutions of various functional groups on the core of the phenazine ring structure. Thus, Yuan et al. demonstrated with Pseudomonas chlororaphis defective or overproducing strains, that phenazine production improved the efficiency in increasing wheat salt tolerance. The fungal Trichoderma strain isolated by Tseng et al. acting as biocontrol effector (see above) could grow on high $\mathrm{NaCl}$ or mannitol concentrations and improved salt tolerance of colonized A. thaliana.

With respect to soil nutrient conditions, results obtained by Cardoso et al. showed the potential ability of the PGPB strain Bacillus cereus UFRABC40 to promote the growth performance of coconut seedlings under decreased application of inorganic fertilizers. Seedling treatments by $100 \%$ chemical fertilizer NPK or $50 \%$ NPK together with B. cereus indicated that the inoculation increased phytohormone levels (IAA, GA) and leaf gas exchange (by assimilation of $\mathrm{CO}_{2}$, stomatal conductance to water vapor, transpiration and instantaneous carboxylation efficiency). Furthermore, growth parameters and macro- and micronutrient levels were improved. More generally, Wang et al. found a change of bacterial diversity and community together with soil properties and plant functioning during long-term grassland restoration and recovery. The observed changes in soil microbial community were tightly linked to the presence of increased soil $\mathrm{C}$ and $\mathrm{N}$ substrates due to plant growth and diversity. However, whether these bacterial changes improved growth and tolerance of plants to facilitate the recovery of the analyzed grassland ecosystem remains to be further studied.

Finally, the use of $\mathrm{N}_{2}$ fixing bacteria in the field to increase essential oil (EO) quantity and quality of sweet basil was explored by Kordi et al.. These authors found that application of free living $\mathrm{N}_{2}$ fixing bacteria Azospirillum brasilense and Azotobacter chroococcum together with $50 \%$ of regular chemical fertilizer enhanced EO quantity and quality, more than when intercropped with maize plants.

The review concerns orchids being among the most endangered in the plant kingdom. Lack of endosperm in their seeds renders orchids to depend on nutrients provided by orchid mycorrhizal fungi (OMF) for seed 
germination and seedling formation in the wild ( $\mathrm{Li}$ et al., 2021). Zhao et al. presented a new technology using seed germination-promoting OMF coming from roots or seeds of orchid plants to be used for reintroduction of orchids in their natural habitat.

In conclusion, this Research Topic, by putting together different beneficial and nutritional aspects affected by a diversity of microorganisms, tries to pave the way for future research about their role in plant mineral nutrition linked to ISR aiming finally in their better use and involvement in a more sustainable and environmentally friendly agriculture.

\section{REFERENCES}

Cameron, D. D., Neal, A. L., van Wees, S. C., and Ton, J. (2013). Mycorrhizainduced resistance: more than the sum of its parts? Trends Plant Sci. 18, 539-545. doi: 10.1016/j.tplants.2013.06.004

de Souza, R., Ambrosini, A., and Passaglia, L. M. (2015). Plant growth-promoting bacteria as inoculants in agricultural soils. Genet. Mol. Biol. 38, 401-419. doi: 10.1590/S1415-475738420150053

De Vrieze, M., Varadarajan, A. R., Schneeberger, K., Bailly, A., Rohr, R. P., Ahrens, C. H., et al. (2020). Linking comparative genomics of nine potato-associated Pseudomonas isolates with their differing biocontrol potential against late blight. Front. Microbiol. 11:857. doi: 10.3389/fmicb.2020.00857

Guzmán-Guzmán, P., Porras-Troncoso, M. D., Olmedo-Monfil, V., and Herrera-Estrella, A. (2019). Trichoderma species: versatile plant symbionts. Phytopathology 109, 6-16. doi: 10.1094/PHYTO-07-18-0218-RVW

Kumar, A., Singh, S., Gaurav, A. K., Srivastava, S., and Verma, J. P. (2020). Plant growth-promoting bacteria: biological tools for the mitigation of salinity stress in plants. Front. Microbiol. 11:1216. doi: 10.3389/fmicb.2020.01216

Kupferschmied, P., Maurhofer, M., and Keel, C. (2013). Promise for plant pest control: root-associated pseudomonads with insecticidal activities. Front. Plant Sci. 4:287. doi: 10.3389/fpls.2013.00287

Li, T., Yang, W., Wu, S., Selosse, M.-A., and Gao, J. (2021). Progress and prospects of mycorrhizal fungal diversity in orchids. Front. Plant Sci. 12:646325. doi: 10.3389/fpls.2021.646325

Lucena, C., Porras, R., Romera, F. J., Alcántara, E., García, M. J., and Pérez-Vicente, R. (2018). Similarities and differences in the acquisition of Fe and P by dicot plants. Agronomy 8:148. doi: 10.3390/agronomy8080148

Pieterse, C. M. J., Zamioudis, C., Berendsen, R. L., Weller, D. M., Van Wees, S. C. M., and Bakker, P. A. H. M. (2014). Induced systemic resistance by beneficial microbes. Annu. Rev. Phytopathol. 52, 347-375. doi: 10.1146/annurev-phyto-082712-102340

Rivero, J., Lidoy, J., Llopis-Giménez, Á., Herrero, S., Flors, V., and Pozo, M. J. (2021). Mycorrhizal symbiosis primes the accumulation of antiherbivore compounds and enhances herbivore mortality in tomato. J. Exp. Bot. 72, 5038-5050. doi: 10.1093/jxb/erab171

\section{AUTHOR CONTRIBUTIONS}

RA, SZ, and CL reviewed and summarized the articles of the Topic. CL wrote the draft. All authors revised and validated the final manuscript.

\section{ACKNOWLEDGMENTS}

The Topic Editors are grateful to all authors who contributed to this Research Topic, to the Reviewers for evaluating their work, and to the Frontiers editorial staff for their guidance and production assistance.

Romera, F. J., García, M. J., Lucena, C., Martínez-Medina, A., Aparicio, M. A., Ramos, J., et al. (2019). Induced systemic resistance (ISR) and Fe deficiency responses in dicot plants. Front. Plant Sci. 10:287. doi: 10.3389/fpls.2019.00287

Verbon, E. H., Trapet, P. L., Kruijs, S., Temple-Boyer-Dury, C., Rouwenhorst, T. G., and Pieterse, C. M. J. (2019). Rhizobacteria-mediated activation of the Fe deficiency response in Arabidopsis roots: Impact on Fe status and signaling. Front. Plant Sci. 10:909. doi: 10.3389/fpls.2019.00909

Verbon, E. H., Trapet, P. L., Stringlis, I. A., Kruijs, S., Bakker, P. A. H. M., and Pieterse, C. M. J. (2017). Iron and immunity. Annu. Rev. Phytopathol. 55, 355-375. doi: 10.1146/annurev-phyto-080516-03 5537

Zamioudis, C., Korteland, J., Van Pelt, J. A., van Hamersveld, M., Dombrowski, N., Bai, Y., et al. (2015). Rhizobacterial volatiles and photosynthesis-related signals coordinate MYB72 expression in Arabidopsis roots during onset of induced systemic resistance and iron-deficiency responses. Plant J. 84, 309-322. doi: $10.1111 /$ tpj. 12995

Conflict of Interest: The authors declare that the research was conducted in the absence of any commercial or financial relationships that could be construed as a potential conflict of interest.

Publisher's Note: All claims expressed in this article are solely those of the authors and do not necessarily represent those of their affiliated organizations, or those of the publisher, the editors and the reviewers. Any product that may be evaluated in this article, or claim that may be made by its manufacturer, is not guaranteed or endorsed by the publisher.

Copyright (c) 2021 Lucena, Zimmermann, Wang and Aroca. This is an open-access article distributed under the terms of the Creative Commons Attribution License (CC $B Y)$. The use, distribution or reproduction in other forums is permitted, provided the original author(s) and the copyright owner(s) are credited and that the original publication in this journal is cited, in accordance with accepted academic practice. No use, distribution or reproduction is permitted which does not comply with these terms. 